\title{
Publisher Correction: CRISPR inactivation by integration
}

Joseph Bondy-Denomy (i)

Correction to: Nature Microbiology https://doi.org/10.1038/s41564-021-01007-6, published online 24 November 2021.

In the version of this article initially published, an error appeared in the lower-right section of Fig. 1, where five crRNA strands appeared to the right of the single lilac crRNA now shown. They have been removed in the corrected version of the figure.

The changes have been made to the online version the article.

Published online: 8 December 2021

https://doi.org/10.1038/s41564-021-01037-0

(C) Springer Nature Limited 2021 